\title{
UNUSUAL COLOBOMA OF THE OPTIC NERVE ENTRANCE*
}

\author{
BY \\ CHRISTOPHER PEDLER \\ Department of Pathology, Institute of Ophthalmology, University of London
}

Developmental anomalies of the eye in the region of the optic disc are commonly divided into two groups: those in which failure of coaptation occurs within the optic nerve sheath and those showing lesions of the peripapillary choroid or sclera. With the exception of the small holes sometimes seen on the anterior surface of the optic disc, which are probably related to aberrant formation of Bergmeister's papilla, the first type is much the less frequent of the two, and probably all the anomalies in the second group are formed by imperfect closure of the foetal fissure with the result that they appear on a line running inferiorly from the rim of the optic disc to the equator.

The following report describes a coloboma of the optic nerve entrance of unusual configuration which does not belong to either group.

\section{Case Report}

A male child aged 13 months was first seen because of a left convergent squint, and on subsequent examination, a mass was discovered in the neighbourhood of the optic disc. Under regular observation at intervals for 6 months, no significant changes were noted. In the seventh month after the first presentation, however, it was the opinion of a number of observers that the lesion was increasing in size and now resembled a glioma of the retina. In view of this opinion and the fact that the optic nerve-head was visibly involved, the eye was removed.

\section{Pathology}

Macroscopical Appearances.-The eye was of normal shape and size and the only external abnormality noted was a symmetrical thickening of the optic nerve at its junction with the posterior sclera. There was no constriction at this point such as that typically associated with a glioma of the optic nerve. No retro-orbital cyst was present and no evidence of the foetal fissure could be seen with the unaided eye.

The eye was opened by incision in the sagittal plane horizontally, when the optic nerve was seen to be surrounded by an approximately conical mass of firm greyish white tissue containing scattered pigment. The optic disc appeared to protrude forwards into the vitreous space. The pattern of the retinal vessels appeared macroscopically normal and no further abnormalities were noted at this stage.

Microscopical APPEARANCES.-The eye was embedded in celloidin and sections were cut at a thickness of $12 \mu$ through the entire disc region in order to delineate the total extent of the lesion and to understand its general shape. 
Apart from the main lesion, oedema of the equatorial choroid and some evidence of posterior synechiae on the anterior surface of the lens, the structures of the eye were within normal limits histologically. No evidence was seen of other conditions occasionally associated with colobomata of the optic nerve entrance (persistent hyaloid artery, opaque nerve fibres, lens opacities, or other colobomata of the choroid, macula, or iris).

The general arrangement of the structures involved in the lesion is seen in Fig. 1, which is a horizontal section in the sagittal plane passing through the midline of the optic nerve in its longitudinal axis. It will be noted that the optic disc and cribriform plate are displaced posteriorly (A, Fig. 1). Normally, the plane of the discontinuous connective tissue forming the lamina cribrosa is approximately in line with the level of the sclera (Fig. 2, opposite), whereas in this case it was found at a point outside the external line of the globe. Furthermore, it was bowed posteriorly. There was therefore no true optic disc in the normal position and what must have appeared clinically as the physiological pit (B, Fig. 1) was in fact a conical hole formed by the inner surfaces of the retina becoming continuous as the optic disc was thrust backwards by the developing intra-ocular pressure. Adjacent to the false disc on the medial side, there was, in addition, a mass of disorganized and heavily gliosed retinal tissue (C, Fig. 1). On low-power microscopy, this appeared to be a simple retinal fold, but high-power examination revealed actual cellular proliferation probably originating from the two nuclear layers; together with numerous psammoma bodies, glial cells, and fibroblasts. The immediately adjacent

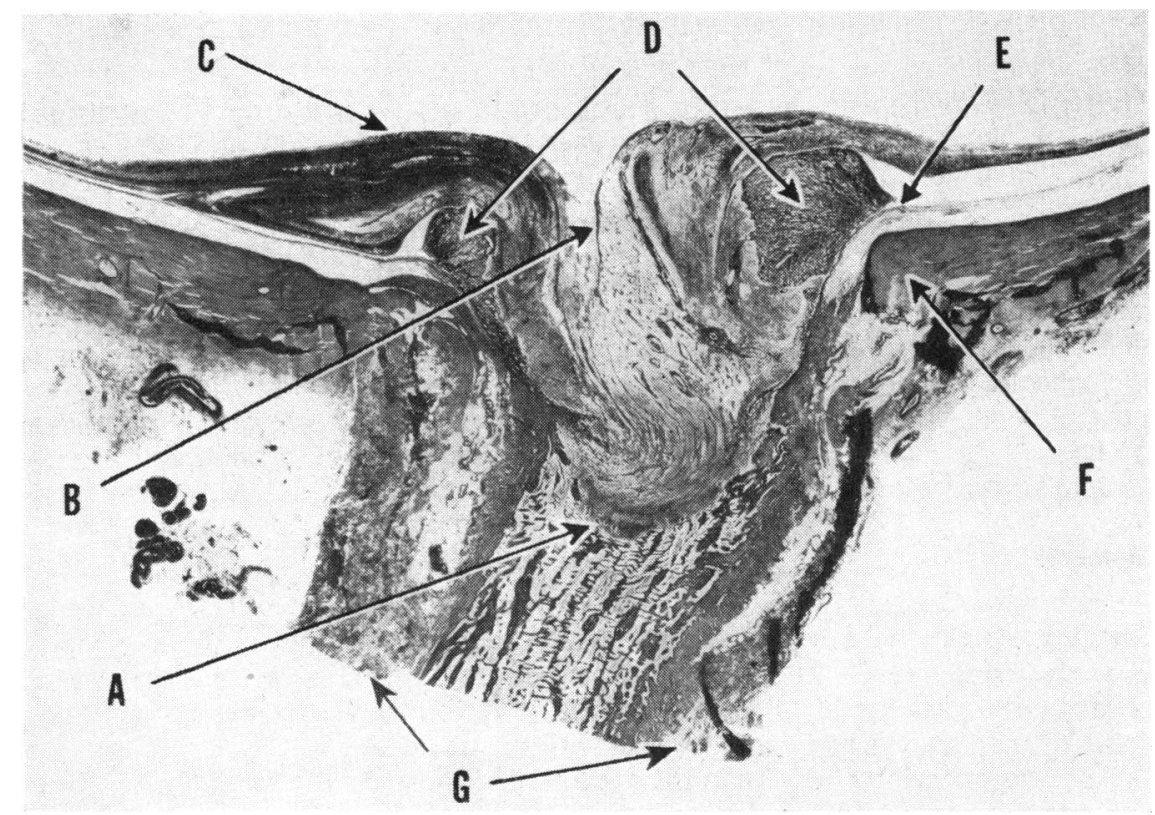

FIG. 1.-Horizontal section in the sagittal plane through the midline of the optic nerve. Wilder's silver diamino-hydroxide. $\times 15$.
A. Displaced lamina cribriformis.
B. False physiological pit, formed by coapted internal surface of prolapsed retina.
C. Retinal fold containing aberrant retinal tissue.
D. Annular glial mass overlying scleral dehiscence.
E. Normal choroid prolapsing through coloboma.
F. Unfused scleral extremity.
G. Thickened optic nerve sheath. 
Fig. 2.-A representation of the normal relations between sclera, choroid, retina, and optic nerve at the optic disc.

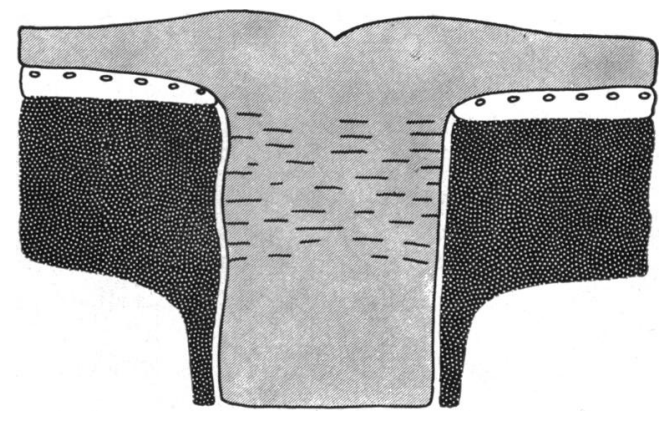

retina showed two abnormalities: a marked gliosis of the stratum opticum, and an attenuation of the outer nuclear layer, together with a localized absence of the visual cells. The remainder of the retina including the central area was normally formed, but had undergone generalized atrophy, together with a slight replacement gliosis of the inner cell layers. There was, in addition, a paucity of retinal vessels.

The choroid, with the exception of the peripheral oedema already mentioned, was normally formed, the vessels, stroma, pigment cells, and retinal pigment epithelium were all present and normal; the only intrinsic abnormality noted was a generalized thinning of Bruch's membrane. At its posterior extremity, however, the entire thickness of the choroid had prolapsed through the coloboma to become continuous with the imperfectly formed optic nerve sheath (E, Fig. 1).

The most severely affected tissue in this case was the sclera. Posteriorly, it had completely failed to fuse with the optic nerve and its sheath, with the result that there was an annular space surrounding the optic nerve as it leaves the globe. This is best seen in Fig. 3 (also F, Fig. 1), where the end of the unfused sclera on one side is shown with the

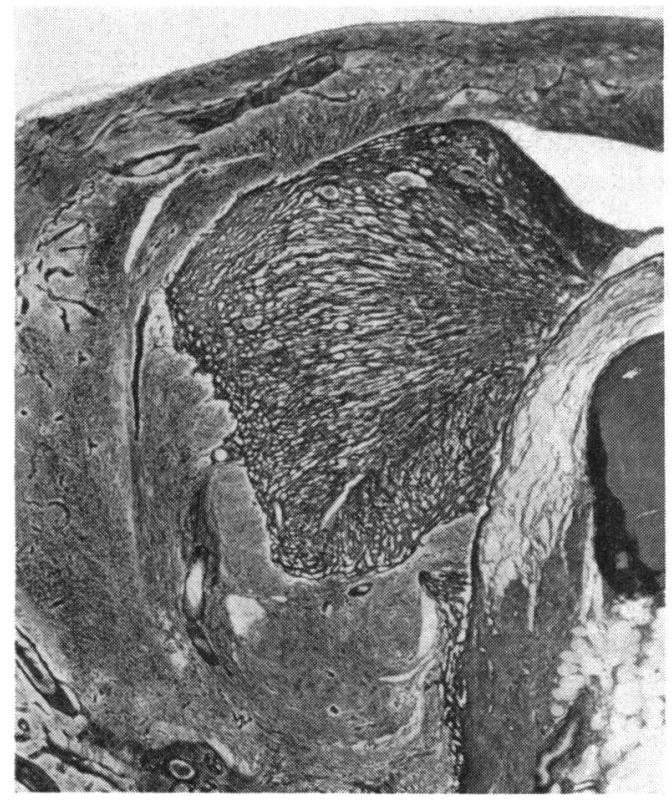

FIG. 3.-To show normal choroid prolapsing through the coloboma. The choroid passes downwards between the unfused end of the sclera on the right and the glia in the centre, to become continuous with a mass of fat and immature collagen. Wilder's stain. $\times 67$. 
choroid passing through the opening to become continuous with the imperfectly differentiated collagen surrounding the optic nerve. The fact that this space is annular and not a unilateral cleft was established by the examination of a large number of sections cut through the entire disc region. In addition to the basic failure of coaptation described, the sclera also showed a marked lack of mature differentiation on its external aspect; the normally precise outline of its outer surface being replaced by unorientated spindle cells arranged in a coarse reticular form resembling embryonic mesenchyme. The optic nerve sheath was similarly affected and in addition to containing localized aggregations of fat, presented a loose areolar appearance, again suggestive of undifferentiated mesodermal tissue (G, Fig. 1).

The final component of this unusual lesion was formed by an annular mass overlying the scleral dehiscence separating the peripapillary retina from the prolapsed choroid (D, Fig. 1). It was composed of compact aggregations of glial cells, fibroblasts, vessels, and pigment-containing cells, set in a dense matrix of reticulin. The probability is that it originated from the retina rather than the choroid or sclera since, in some sections, there was a plane of cleavage between its outer surface and the choroid, whereas its inner aspect was intimately continuous with the retina.

Finally, the optic nerve posterior to the displaced lamina cribrosa was grossly abnormal. The normally regular glial compartments separating the axon bundles were ruptured and distorted so that the nerve fibres pursued a totally irregular course.

\section{Discussion}

The first indication of alignment and orientation of the paraxial mesoderm surrounding the expanding optic vesicle is apparent at approximately the $18-\mathrm{mm}$. stage and from this point onwards, scleral maturation proceeds until by the 150 to $180-\mathrm{mm}$. stage it is virtually complete. Collagen lamellae are first seen at the future insertion of the rectus muscles and, from this point, development proceeds forwards to the limbal region and posteriorly to the optic disc. If, therefore, any part of the sclera is to show localized congenital abnormality it is most likely to occur posteriorly, provided that the remainder matures normally. The choroid, although developing from mesoderm immediately adjacent to the sclera, undergoes slightly earlier differentiation; the chorio-capillaris is apparent at the $65-\mathrm{mm}$. stage, and all layers are recognizable at the $140-\mathrm{mm}$. stage. It is therefore not surprising that almost fully-formed choroid protrudes into the coloboma. It is, of course, impossible to be certain exactly when the intra-ocular pressure first develops, it could conceivably begin at an early stage, for the foetal fissure closes at $14 \mathrm{~mm}$. There is however no differentiated ciliary body at this time and the canal of Schlemm is not seen clearly before the 60 to $65-\mathrm{mm}$. stage. Even if the intra-ocular pressure developed at a late stage of intra-uterine life, say at the $250-\mathrm{mm}$. stage, there would probably still be sufficient time for the gross displacements present in this case to be formed, for it may equally well be that although the train of events was begun in utero by the scleral failure, the actual deformity evolved gradually during the post-natal period as a result of the outward force exerted by the intra-ocular pressure. Furthermore, the scleral lesion itself may not have been the initial fault; 
the primary abnormality may have occurred in the optic cup. For the sclera is not a self-determining structure, but exhibits the phenomenon of double assurance, and although the optic cup is not the sole determinant for the sclera, since a nodule of scleral tissue may develop in cases of anophthalmia, some support for the possibility is provided in this case by the presence of aberrant retinal tissue,

To summarize, therefore, it is impossible to be certain of the precise order of intra-uterine events preceding the formation of this curious lesion. Most probably, the basic defect was a failure of maturation in the posterior sclera, possibly initiated by a derangement of the optic cup, so that normal fusion failed to occur and the peripapillary retina, optic disc, lamina cribrosa, and posterior choroid were all driven posteriorly through the resultant hiatus (Fig. 4).

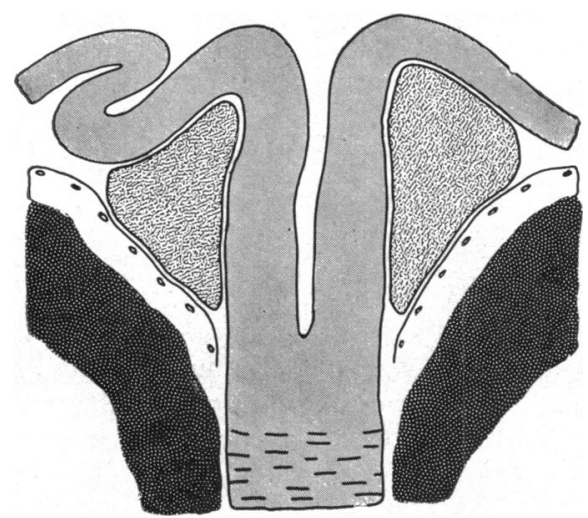

FIG. 4.-To summarize the displacements present in this case: the sclera and optic nerve sheath have failed to form properly, with the result that the lamina cribrosa, retina, and choroid have been displaced posteriorly. This has caused the retina to invaginate, and to form a false physiological pit together with a mass of glial tissue interposed between the scleral hiatus and the retina.

I should like to thank Mr. Norman Ashworth for permission to publish the clinical details of this case which was under his care. 